\title{
Thermal roughening of a solid-on-solid model with elastic interaction
}

\author{
Frank Gutheim, Heiner Müller-Krumbhaar, and Efim Brener \\ Forschungszentrum Jülich, D-52425 Jülich, Germany
}

Vladimir Kaganer

Paul-Drude-Institut für Festkörperelektronik, Hausvogteiplatz 5-7, D-10117 Berlin, Germany

(Received 6 September 2002; revised manuscript received 21 January 2003; published 6 May 2003)

\begin{abstract}
We analyze the effects of a long-ranged interaction between surface defects on thermal roughening within the framework of a solid-on-solid model of a crystal surface by means of Monte Carlo simulation. Below roughening the interaction can be understood in terms of a repulsive step-step interaction, which is modeled by elastic dipoles located on sites adjacent to the steps. In order to reduce the computational effort involved in calculating interaction energy based on long-ranged potentials, we employ a multigrid scheme. As a result of the long-range character of the repulsive step interaction, the roughening temperature increases drastically compared to a system with short-range cutoff as a consequence of anticorrelations between surface defects.
\end{abstract}

DOI: 10.1103/PhysRevB.67.195404

PACS number(s): 68.35.Rh

\section{INTRODUCTION}

At low temperatures crystal surfaces are known to assume the shape of a plane facet. With increasing temperature fluctuations gradually contribute a nonzero thickness to the initially flat facet. This surface thickness finally diverges at a finite temperature, the roughening temperature, where the order of the facet is lost completely. This transition can be described by a set of renormalization group equations first analyzed by Kosterlitz and Thouless. ${ }^{1}$ Because of its unusual properties and the relation to the two-dimensional Coulomb gas, ${ }^{2}$ this roughening transition has attracted substantial attention. ${ }^{3-6}$

Various discrete solid-on-solid (SOS) models have been shown to undergo this type of transition. Most of these models incorporate local interactions, at most next-nearestneighbor interactions. Within some of these models a transition involving in-plane disorder is possible, usually referred to as preroughening. ${ }^{7-14}$

Interaction of surface defects by means of elastic deformation of the crystal, however, is of a long-ranged nature and has apparently not been previously studied in the context of roughening. Leaving the matter of preroughening aside, we will try to elucidate the effects of long-range elastic interactions on the roughening process.

The paper is organized as follows. First we will introduce elastic interaction between surface defects and suggest some simplifications to make the problem tractable. Then we present the details of our discrete solid-on-solid model allowing for long-range step interaction. We will show the results of our extensive Monte Carlo simulations and interpret the effects.

\section{STEP INTERACTION}

The elastic step interaction on the surface of a semiinfinite crystal can be described in terms of elastic force dipoles located at the step edges. ${ }^{15-18}$ Knowing the Green function $G_{i j}$ for an infinite elastic half-space one is able to calculate the elastic displacement field $u_{i}(\mathbf{r})$ at a position on the crystal surface indicated by a two-dimensional in-plane vector $\mathbf{r}$ with components $r_{x}, r_{y}$ from a given force density $f_{i}(\mathbf{r})$ :

$$
u_{i}(\mathbf{r})=\int d^{2} r^{\prime} G_{i j}\left(\mathbf{r}-\mathbf{r}^{\prime}\right) f_{j}\left(\mathbf{r}^{\prime}\right) .
$$

The indices $i, j$ correspond to directions $x, y$, and $z$. Repeated indices are implicitly summed over. The elastic energy $E_{\mathrm{el}}$ becomes

$$
\begin{aligned}
E_{\mathrm{el}} & =-\int d^{2} r u_{i}(\mathbf{r}) f_{i}(\mathbf{r}) \\
& =-\iint d^{2} r d^{2} r^{\prime} G_{i j}\left(\mathbf{r}-\mathbf{r}^{\prime}\right) f_{j}\left(\mathbf{r}^{\prime}\right) f_{i}(\mathbf{r}) .
\end{aligned}
$$

Using the fact that forces $f_{i}(\mathbf{r})$ are present only in the vicinity of a step and that the monopole moment at the step vanishes, we can rewrite the energy using force dipole densities $q_{i k}(\mathbf{r})$ as the next term in a multipole expansion

$$
E_{\mathrm{el}} \approx \iint d^{2} r d^{2} r^{\prime} q_{j k}\left(\mathbf{r}^{\prime}\right) q_{i l}(\mathbf{r}) \partial_{k} \partial_{l} G_{i j}\left(\mathbf{r}-\mathbf{r}^{\prime}\right) .
$$

Using symmetry arguments one can determine two types of force dipoles that are considered to be present at a step. ${ }^{16}$ One type involves in-plane forces perpendicular to the step, the other arises from forces orthogonal to the crystal surface. Due to the structure of the Green function, dipole tensors involving forces orthogonal to the surface show a behavior different from those involving only in-plane forces. ${ }^{16,18}$ The former lead to attractive or repulsive interaction depending on the signs of the steps, the latter produce a signindependent behavior, which is strictly repulsive. There are materials $^{19,20}$ where the sign-dependent contributions are small compared to the step repulsion caused by in-plane forces, and we will restrict our model to the case where we can neglect sign dependence of the steps.

In the case of isotropic linear elasticity the half-space elastic Green function $G_{i j}(\mathbf{r})$ can be written in a simple form: ${ }^{15}$ 


$$
G_{i j}(\mathbf{r})=\frac{1+\sigma}{\pi E} \frac{1}{r}\left\{(1-\sigma) \delta_{i j}+\sigma \frac{r_{i} r_{j}}{r^{2}}\right\},
$$

where $i$ and $j$ are now restricted to in-plane coordinates and $r=|\mathbf{r}|$. The only two material parameters involved in Eq. (4) are the dimensionless Poisson ratio $\sigma$ and the Young's modulus $E$.

For a step stretching in the $y$ direction one would assume the force dipole tensor at the step to be of the type $q_{i j}$ $\sim \delta_{i x} \delta_{j x}$. This means that the interaction between two line elements will depend on their orientation.

In the case of two parallel steps, a distance $d$ in the $x$ direction apart, the interaction energy density $w$ (per area squared) can be computed by evaluating the integrand from Eq. (3) for two interacting force dipoles of the type $q_{i j}$ $=\delta_{i x} \delta_{j x}$. It is given by

$$
w(r, \varphi)=\gamma\left[\frac{3 \cos \varphi-1}{r^{3}}+\frac{\sigma}{1-\sigma} \frac{2+15 \cos ^{4} \varphi-15 \cos ^{2} \varphi}{r^{3}}\right],
$$

where $\varphi$ denotes the angle between the radius vector $\mathbf{r}$ and the orientation of the dipole forces, which is given by $\varphi$ $=\arctan (\Delta y / d)$, and $\Delta y$ is the distance between the dipoles in the $y$ direction. The factor $\gamma$ is given by

$$
\gamma=\frac{1-\sigma^{2}}{\pi E} Q^{2}
$$

where $Q$ is the dipole moment per unit length of the step.

Integrating the energy density for a configuration with two parallel steps at distance $d$, we state that the energy per unit length of the line is just

$$
\widetilde{W}=4 \gamma \frac{1}{d^{2}}-2 \gamma \frac{1}{\varepsilon^{2}} \frac{1-2 \sigma}{1-\sigma}
$$

where the interaction was limited to distances greater than $\varepsilon$. Note that the second term, which contributes to line energy, is negative for all possible Poisson ratios $-1 \leqslant \sigma \leqslant 1 / 2$.

In order to make another simplification of the step-step interaction we compare the above result to the case of a scalar $w \sim 1 / r^{3}$ interaction associated with isotropic dipoles $q_{i j} \sim \delta_{i j}$

$$
\widetilde{W}_{\text {scalar }}=4 \gamma \frac{1}{d^{2}}+2 \gamma \frac{1}{\varepsilon^{2}}
$$

from which we conclude that the only difference in this specific geometry is a change in the line energy, which is mainly due to contributions from short range interactions.

Because we aim at showing the effect of long-range interactions on the thermal roughening process, we neglect the angular dependence completely and assume that the dipole moments are isotropic. This leads to a simple isotropic $1 / r^{3}$ interaction between force dipoles. Furthermore, this ensures that the elastic contribution to the step energy is positive.

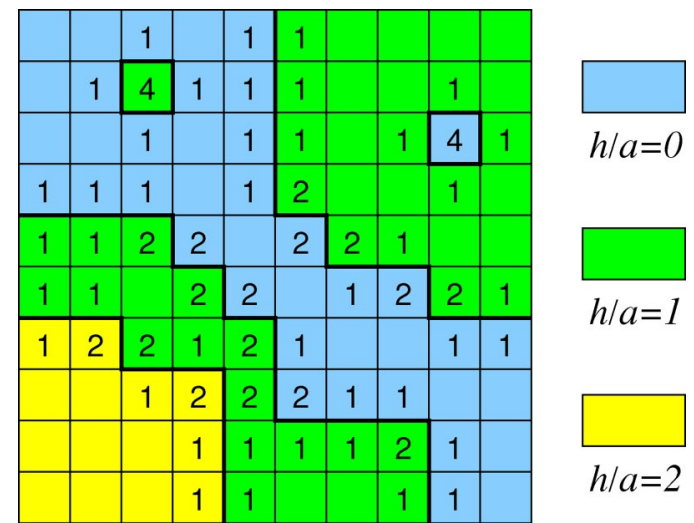

FIG. 1. The number of dipole charges assigned to a lattice site is proportional to the accumulated absolute height difference corresponding to Eq. (10).

\section{MODEL DESCRIPTION}

Within the framework of a solid-on-solid model we describe the crystal surface by a simple height field $h_{i}$ of integer multiples of the lattice constant $a$. Like in a common SOS model, overhangs are forbidden. Instead of the usual surface energy term (summation over nearest neighbors)

$$
E_{\text {surf }}=\frac{J}{2 a^{\alpha}} \sum_{\langle i, j\rangle}\left|h_{i}-h_{j}\right|^{\alpha}
$$

with coupling constant $J$ and $\alpha=1,2$ for the absolute solidon-solid (ASOS) model and the discrete Gaussian solid-onsolid (DGSOS) model respectively, we define an elastic step interaction by introducing a field of elastic dipole charges $q$. To every lattice site a dipole charge $q_{k}$ proportional to the number of height differences to the four neighboring sites is assigned, i.e., site $k$ carries a number of

$$
q_{k}=\frac{1}{a} \sum_{\langle i, j\rangle}\left|h_{i}-h_{j}\right| \delta_{i k}
$$

charges. Figure 1 gives an example how charges are assigned to a simple height field configuration. The elastic dipole charges interact, in consequence of Eq. (8), via a modified $r^{-3}$ interaction potential $\Psi_{p_{\max }}(r)$,

$$
\Psi_{p_{\max }}(r)=\min \left((a / r)^{3}, p_{\max }\right),
$$

where $r$ is the in-plane distance between two lattice sites measured in units of the lattice constant and $p_{\max }$ is a number limiting the interaction potential in vicinity of $r=0$. For $a / r>\sqrt[3]{p_{\max }}$ the potential is given by $\Psi_{p_{\max }}(r)=(a / r)^{3}$, otherwise the potential is just constant, i.e., $\Psi_{p_{\max }}(r)=p_{\max }$. This gives rise to the elastic energy

$$
E_{\mathrm{el}}=\frac{w_{\mathrm{el}}}{2} \sum_{i, j} q_{i} q_{j} \Psi_{p_{\max }}\left(r_{i j}\right),
$$

where $r_{i j}$ is the distance between lattice sites $i$ and $j$ and $w_{\mathrm{el}}$ can be adjusted to give the desired interaction strength. Note that the case $i=j$ is not excluded from the summation. 
Later we also limit the range of interaction. For this purpose we introduce a cutoff potential $\Psi_{p_{\max }, l}$ with cutoff length $l$,

$$
\Psi_{p_{\max }, l}(r)=\left\{\begin{array}{lll}
\Psi_{p_{\max }}(r) & \text { if } & r \leqslant l \\
0 & \text { if } & r>l
\end{array}\right.
$$

which vanishes for distances greater than $l$. For two straight steps of length $L$ with distance $d$ this elastic energy contribution consists of the self-energies of the steps and the expected $\sim d^{-2}$ step interaction term

$$
E_{\mathrm{int}} \approx 8 w_{\mathrm{el}} \frac{L a}{d^{2}},
$$

for large distances $d \gg a$.

By modifying $p_{\max }$ the self-energy contribution of a straight step can be adjusted to the desired line energy, independently of the step-step interaction amplitude. For given $p_{\max }$ the relative amplitude of line energy and step-step interaction is fixed and we can concentrate on the crossover from a local to a long-range model depending on the cutoff length $l$, which is studied using the interaction potentials $\Psi_{1, l}$ where $p_{\max }=1$. Later, however, other relative amplitudes are studied for the potential $\Psi_{p_{\max }}$ without cutoff.

The simulation is carried out on a square lattice of size $(L / a)^{2}=64 \times 64$ to $128 \times 128$. In order to calculate the difference in energy for every metropolis Monte Carlo trial, we apply a multigrid scheme based on Ref. 21, which has already been applied successfully to submonolayer epitaxy. ${ }^{22}$

This cuts down the computational costs from order $(L / a)^{4}$ to order $(L / a)^{2} \ln (L / a)$ for each time step, which has to be multiplied by an additional factor of $(L / a)^{2}$, for the number of time steps the system needs to equilibrate. Without the use of the multigrid scheme the computational costs would not have permitted system sizes beyond $L / a=25$. Still, the system size $L / a \leqslant 128$ is rather restricted and we are aware that the results should be accounted as qualitative rather than quantitative. However, computations on the DGSOS and ASOS models at $L / a=128$, which we did for comparison, give transition temperatures $k_{\mathrm{B}} T_{\mathrm{R}} \approx 1.5 \mathrm{~J}$ and $k_{\mathrm{B}} T_{\mathrm{R}} \approx 1.25 J$ respectively, where $k_{\mathrm{B}}$ is the Boltzmann constant. These values agree reasonably well with known results. $^{23}$

\section{RESULTS AND DISCUSSION}

\section{A. Height correlation function}

We determine the roughening temperature $T_{\mathrm{R}}$ from the behavior of the height-height correlation function. Below roughening, $T<T_{\mathrm{R}}$, the interface is macroscopically flat, i.e., the height-height correlation function

$$
G(r)=\frac{\left\langle[h(0)-h(r)]^{2}\right\rangle}{a^{2}}
$$

approaches a finite value in the limit $r \rightarrow \infty$. To be more precise, the correlation length $\xi$ is finite and the interface has a characteristic width. Approaching the transition tempera- ture the correlation length increases and diverges at $T$ $=T_{\mathrm{R}}$. For $T>T_{\mathrm{R}}$ the correlation function $G(r)$ diverges $^{23}$ according to the conventional theory of the roughening transition,

$$
G(r) \sim K(T) \ln \frac{r}{a},
$$

with an amplitude $K(T)$ depending on the temperature. Plotting $G(r)$ vs $\ln (r / a)$, one could determine at what temperature the correlation length $\xi$ diverges and the graphs approach a straight line.

In a finite system with periodic boundary conditions, however, the correlation length $\xi$ cannot exceed the system size $L$, and the height-height correlation function $G(r)$ saturates for $T>T_{\mathrm{R}}$ as well. In order to overcome this finite-size effect, we will use an approach similar to the one used in Ref. 24. In order to keep the argument simple we only consider correlations along the main directions of the lattice and replace $r$ by $x=r_{x}$.

As the limiting behavior of $G(x)$ for periodic boundary conditions has to be a periodic function that behaves like the logarithm for distances $\ll L$, we define a "periodic logarithm" by means of Fourier analysis. In order to avoid the singularity at $\zeta=x / a \rightarrow 0$ we start with

$$
v(\zeta)=\ln [\max (\zeta, 1)]
$$

and the integral Fourier or, using symmetry arguments, the cosine transform

$$
\tilde{v}(k)=\frac{1}{\pi} \int_{0}^{\infty} \cos (k \zeta) v(\zeta) d \zeta
$$

Making use of these Fourier components we define the $L$-periodic function $V_{L}(x)$,

$$
V_{L}(x)=\frac{2 \pi a}{L} \sum_{n=1}^{\infty} \tilde{v}\left(\frac{2 \pi n a}{L}\right) \cos \left(\frac{2 \pi n}{L} x\right) \frac{\sin \left(\frac{2 \pi n a}{L}\right)}{\frac{2 \pi n a}{L}}
$$

which is a discrete back transform averaged over unit distances. For convenience we define

$$
V(x)=V_{L}(x)-V_{L}(L / 2)
$$

and plot $G(x)$ vs $V(x)$. Figure 2 shows the correlation function for the case of the full $1 / r^{3}$ interaction. At a temperature of about $k_{\mathrm{B}} T \approx 9.0 w_{\mathrm{el}}$ the graph becomes straight, indicating the roughening transition. Restricting the elastic dipole charge interaction to distances $\leqslant a$, the graph of the correlation function becomes straight at a lower temperature $k_{\mathrm{B}} T$ $\approx 3.0 w_{\text {el }}$ (see Fig. 3 ).

From the Kosterlitz-Thouless theory of the roughening transition, the slope of the correlation function is expected to assume the universal value $K\left(T_{\mathrm{R}}\right)=2 / \pi^{2}$. Plotting slope versus temperature one can also obtain an estimate of the roughening temperature (see Fig. 4). From this we obtain identical estimates for the two cases with or without cutoff. 


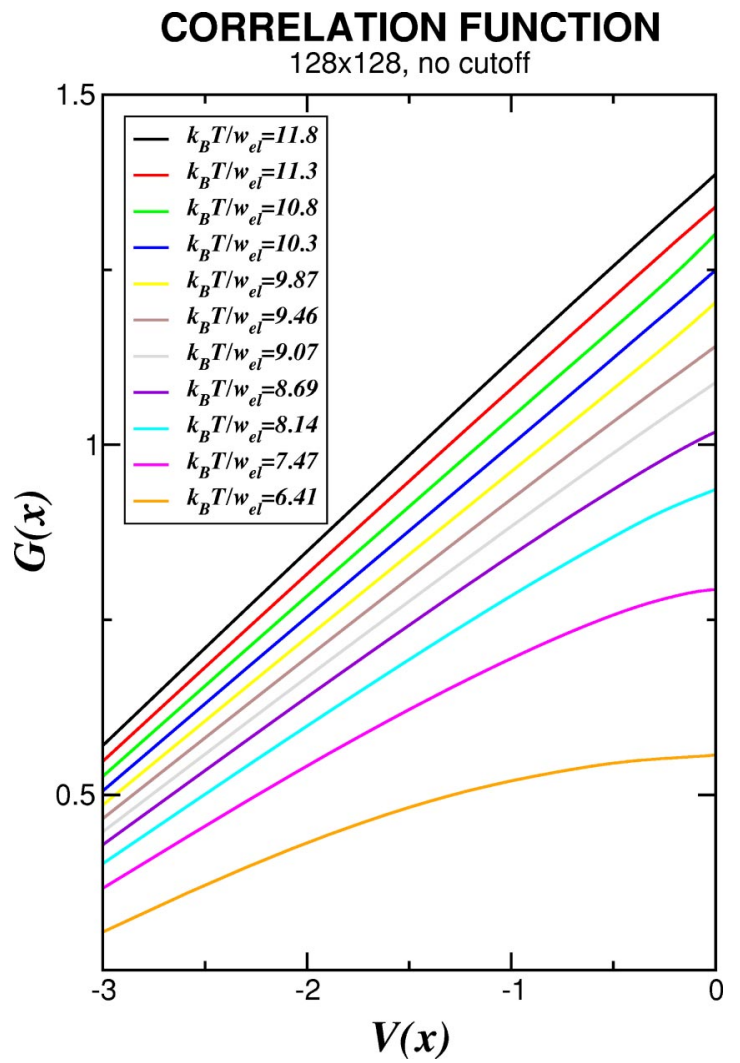

FIG. 2. Height-height correlation function without cutoff. The correlation function saturates for small temperatures and shows logarithmic behavior for $T>T_{\mathrm{R}}$. The first straight line gives an estimate of $k_{\mathrm{B}} T_{\mathrm{R}} / w_{\mathrm{el}} \approx 9.0$.

From this we conclude that the system with long-range interaction has a much higher transition temperature compared to the model with interaction cutoff. The roughening temperature changes by a factor $\sim 3$.

Note that the roughening temperature increases gradually with the cutoff length (see Fig. 5). Even at $l=3 a$ the roughening temperature $k_{\mathrm{B}} T_{\mathrm{R}} \approx 5.8 w_{\mathrm{el}}$ is still well below the value for the infinite-range interaction. The increase of the roughening temperature is not a next-nearest-neighbor effect.

\section{B. Energetic scales}

One might argue that increasing the range of the interaction potential just changes the relevant energetic scale. However, the energetic scales one is usually tempted to think of, i.e., the energy of a straight step or single kinks on such a step, do not change by more than $36 \%$. The straight line energy for a step of length $a$ increases from $w_{1}=1.3 w_{\mathrm{el}}$ at cutoff $l=a$ to $1.7 w_{\mathrm{el}}$ at cutoff $l=\infty$ (see also Fig. 5), and the corresponding kink energy changes from $w_{\mathrm{k}}=2.8 w_{\mathrm{el}}$ to $3.8 w_{\mathrm{el}}$. In the low-temperature regime, the energy of one single adatom on a flat crystal surface is the important energetic scale, which changes from $w_{\mathrm{a}}=8.1 w_{\mathrm{el}}$ to $8.6 w_{\mathrm{el}}$, an increase by no more than $6 \%$.

It should be noted that the main contribution to the change of these energetic scales comes from short-range interactions. Using a cutoff length of $l / a=3$, the straight line and
CORRELATION FUNCTION
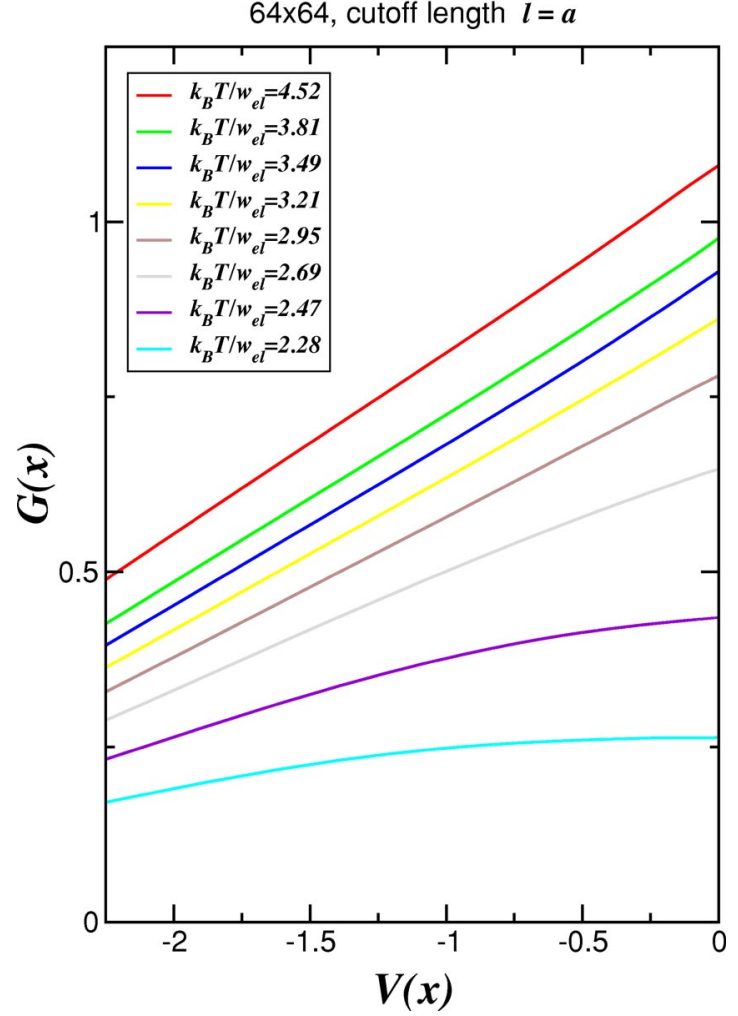

FIG. 3. Height-height correlation function with a cutoff length $l=a$, i.e., only charges on nearest-neighbor sites interact. The first straight line gives an estimate of $k_{\mathrm{B}} T_{\mathrm{R}} / w_{\mathrm{el}} \approx 2.9$.

kink energies are only about $6-7 \%$ below the full potential value, whereas the single adatom defect energy deviates by no more than $0.05 \%$.

From the change of these energetic scales one usually would expect an equal increase of the roughening temperature. One would hesitate, however, to make these changes responsible for an increase of the roughening temperature by

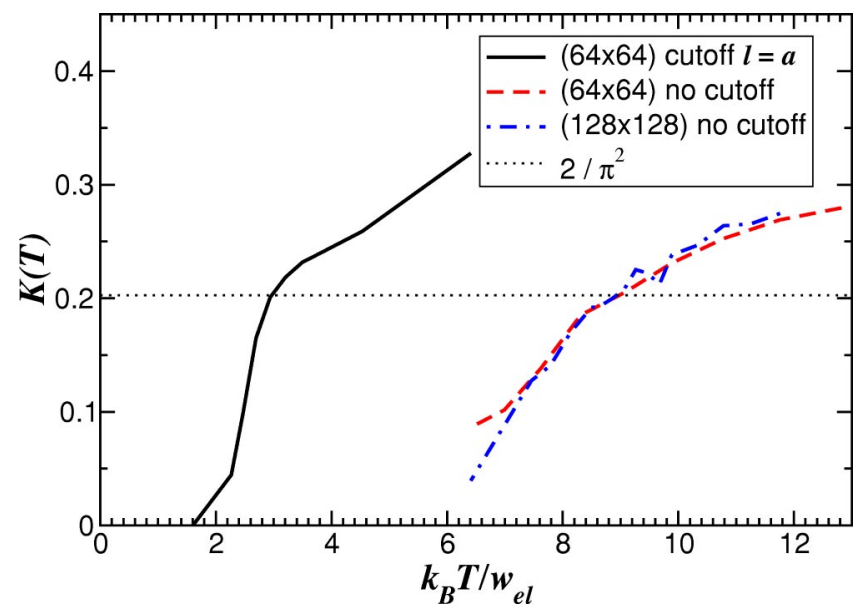

FIG. 4. Slope $K(T)$ vs temperature. Estimation of $T_{\mathrm{R}}$ using the universal value from conventional roughening theory gives $k_{\mathrm{B}} T_{\mathrm{R}}$ $=9.0 w_{\mathrm{el}}$ for infinitely ranged interactions and $k_{\mathrm{B}} T_{\mathrm{R}}=3.0 w_{\mathrm{el}}$ for cutoff length $l=a$. 


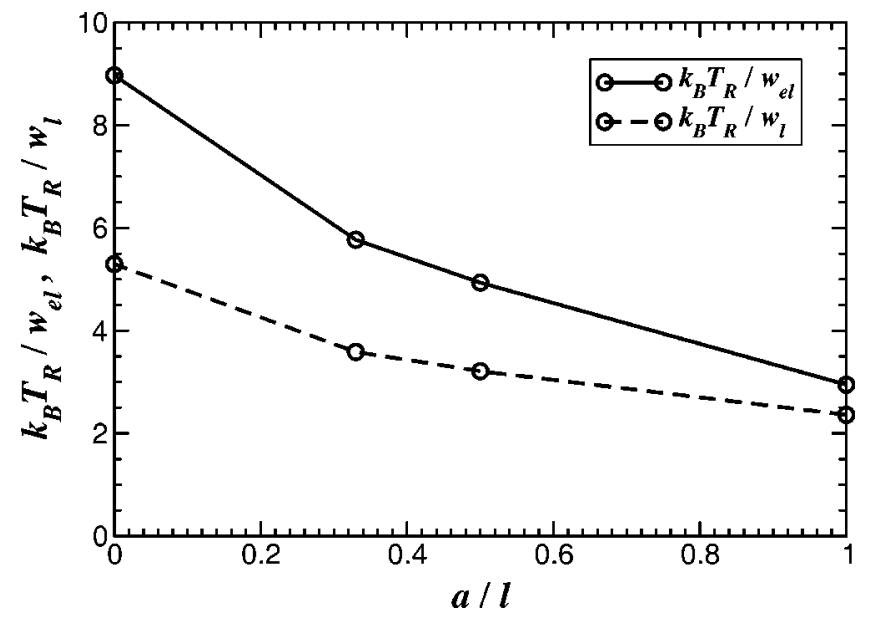

FIG. 5. Scaled roughening temperatures $k_{\mathrm{B}} T_{\mathrm{R}} / w_{\mathrm{el}}$ and $k_{\mathrm{B}} T_{\mathrm{R}} / w_{1}$ vs inverse cutoff length $a / l$. Even at $l / a=3$ the roughening temperature $k_{\mathrm{B}} T_{\mathrm{R}} \approx 5.8 w_{\mathrm{el}}$ is well below the infinite range potential value. $w_{1}$ denotes the energy for a line of length $a$ corresponding to the given potential cutoff $l$.

a factor of $\sim 3$. With a scaling of the roughening temperature by line energy $w_{1}$ rather than $w_{\text {el }}$ (see Fig. 5), a factor $\sim 2$ is left unexplained.

Here we would like to present some arguments based on a high-temperature disordered state, which justifies a larger change of the roughening temperature. Assume for a moment that above the roughening transition the distribution of charges is more or less homogeneous. The energy of an additional dipole charge then relates to the two-dimensional surface integral

$$
\int d^{2} r \Psi(r),
$$

whereas all local energetic scales discussed above depend on effectively one-dimensional charge distributions. Of course the integral has to be replaced by discrete summation if the result is supposed to have any meaning for the model. While the line energy changes by no more than $30 \%$ when changing from $\Psi_{1,1}$ to $\Psi_{1, \infty}$, the two-dimensional sum in the spirit of Eq. (21) changes by a factor of $\sim 2$.

If one assumes that the scale of the roughening temperature is given by Eq. (21), corrections to the asymptotic roughening temperature are of order $a / l$ for $l \gg a$. Naive analytic evaluation of the integral suggests a simple $k_{\mathrm{B}} T_{\mathrm{R}} / w_{\mathrm{el}}$ $\sim 1-2 a / 3 l$ law for the cutoff dependence of the roughening temperature for $\Psi_{1, l}$.

The point is that in the low-temperature regime major energy contributions are local because dipole charges are distributed along steps. For a rough interface, however, the term step does not make any sense and dipole charges can be assumed to be evenly distributed over the whole plane, which effectively changes the dimensionality of energy integration.

\section{Average energy}

Comparing the average energy $E$ of the system computed with and without restriction of the charge interaction range,

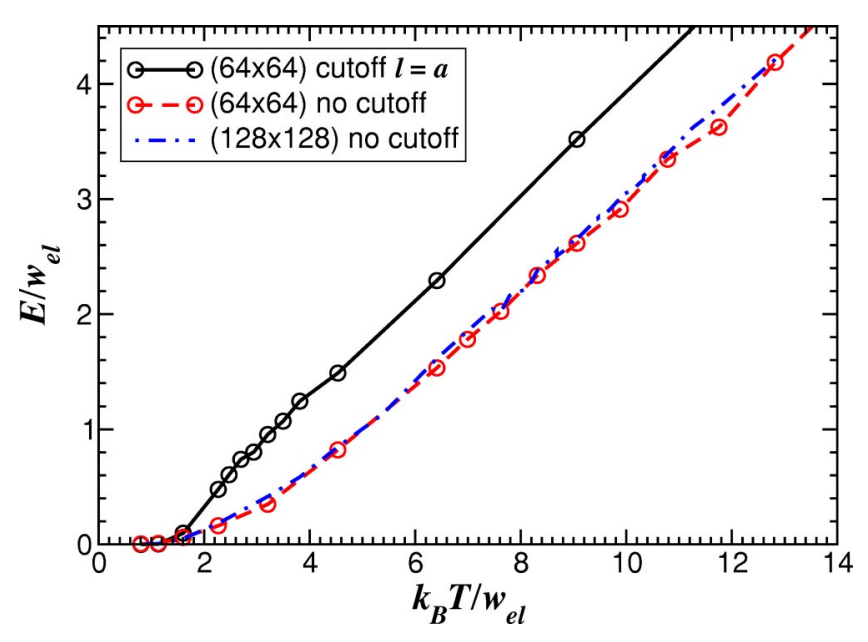

FIG. 6. Average energy per lattice site $E / w_{\text {el }}$ vs temperature $k_{\mathrm{B}} T / w_{\mathrm{el}}$. Average energy for the cutoff potential is strictly higher in comparison to the long-range case.

one clearly sees that the energy for the nonrestricted interaction always stays well below the graph of the restricted system (see Fig. 6). For high temperatures the average energy $E$ goes linear with temperature $T$, indicating that the heat capacity becomes constant.

The range of the interaction potential only affects the behavior below the transition temperature. Above the roughening transition all details of the interaction are combined into one single parameter, the roughening temperature $T_{\mathrm{R}}$. Accordingly the scaled graphs $E / k_{\mathrm{B}} T_{\mathrm{R}}$ vs $T / T_{\mathrm{R}}$ coincide for $T / T_{\mathrm{R}}>1$ (see Fig. 7).

The decrease in average energy of the system using longranged interaction coincides with a smaller number of broken bonds (see Fig. 8). The number of deviations from a facet or the step length is smaller compared to the system with interaction potential cutoff.

\section{Defect correlations}

Restricting the surface height to $\{-a, 0,+a\}$, one may talk about a defect wherever the height deviates from the

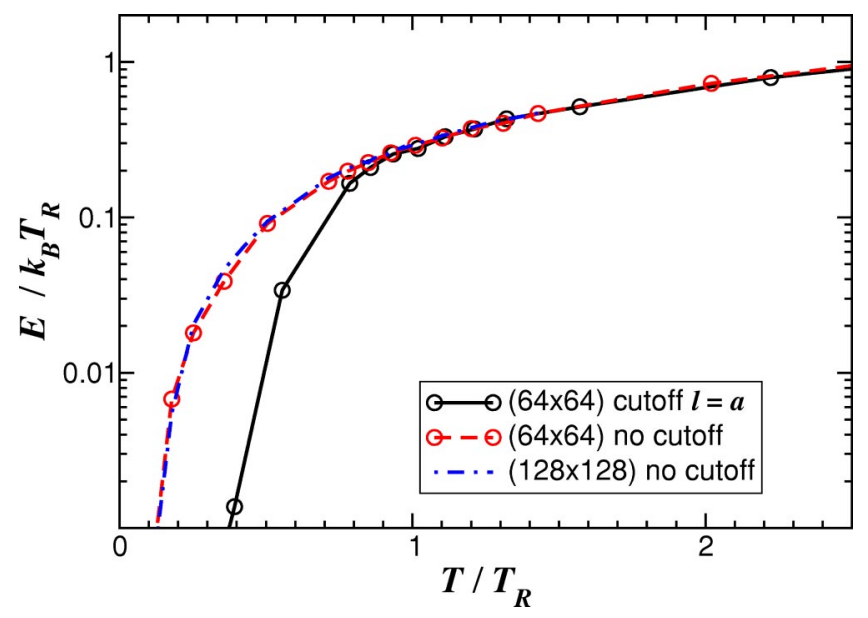

FIG. 7. Scaled average energy per lattice site $E / k_{\mathrm{B}} T_{\mathrm{R}}$ vs scaled temperature $T / T_{\mathrm{R}}$. For $T / T_{\mathrm{R}} \geqslant 1$ the scaled data collapse onto a single graph. 


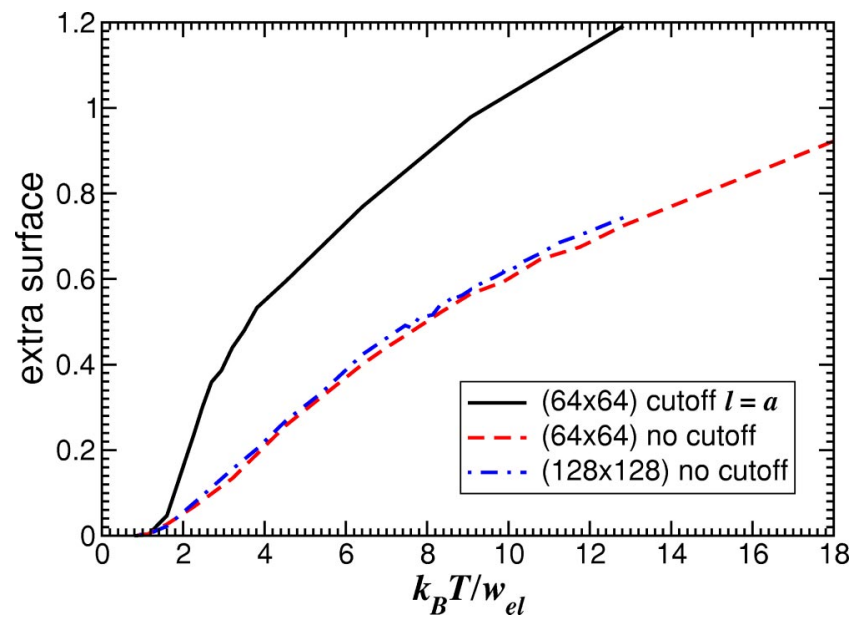

FIG. 8. Extra surface, i.e., number of broken bonds, vs temperature $k_{\mathrm{B}} T / w_{\mathrm{el}}$. Fewer defects are created when no cutoff is used.

average height 0 . Then one can analyze correlation between these defects, i.e., the thermal average of

$$
g_{\text {defect }}(r)=\frac{\left\langle\left[h^{2}\left(\mathbf{r}^{\prime}\right)-h^{2}\left(\mathbf{r}^{\prime}+\mathbf{r}\right)\right]^{2}\right\rangle_{\mathbf{r}^{\prime}}}{\left\langle h^{2}\left(\mathbf{r}^{\prime}\right)\right\rangle_{\mathbf{r}^{\prime}}^{2}} .
$$

Scaled like this, the defect correlation will approach the value 1 for large distances $r$. At low temperatures repulsion between the defects causes the graph to fall below the value of 1 at midrange distances and ends well above the value of 1 at distance $r / a=1$, because contact between equal defects is favored due to what might be called surface or line energy. Increasing the temperature, this repulsion gap will become smaller and vanish eventually.

Figure 9 shows the defect correlation for both the longrange interaction and cutoff for identical temperature. Whereas for the long-range interaction the gap is still present, it has already vanished from the system with cutoff.

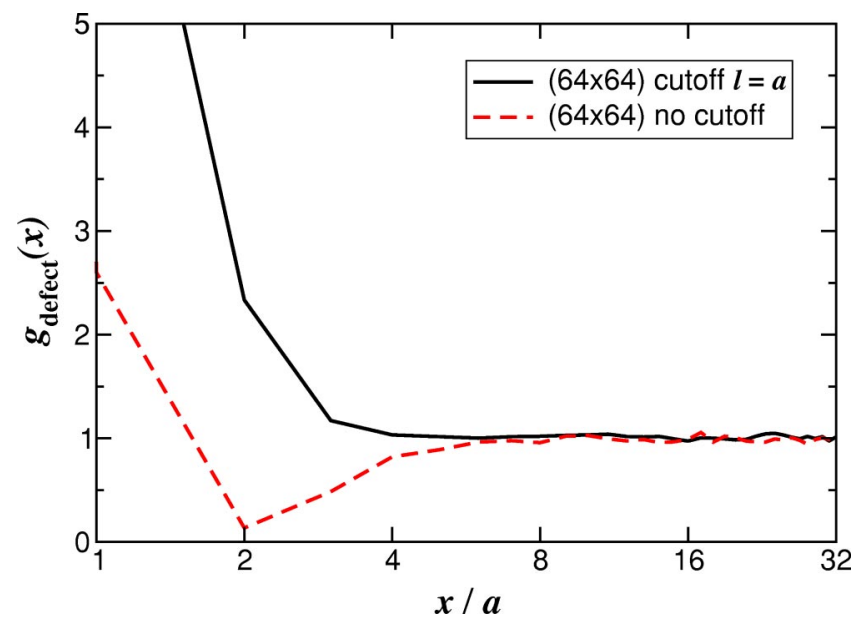

FIG. 9. Defect correlation at $k_{\mathrm{B}} T / w_{\mathrm{el}}=0.5$ for both the longrange interaction and cutoff. The long-range interaction causes a stronger repulsion gap (here near $x / a=2$ ), which means that the defects prefer to be separated. This anticorrelation effect is responsible for a strong decrease in entropy.
The pronounced repulsion gap in the case of the infiniterange interaction means that a single defect or island avoids being close to other defects. This cuts down the number of favorable configurations and thus reduces the entropy contribution to the free energy for a given density of defects $n$.

For the following argument we will assume that the main result is a reduction of entropy by some factor $\alpha<1$, whereas the average energy at given $n$ remains unchanged. In a rather simplified picture we can then write the free energy as $F_{\alpha}=E(n)-T \alpha S(n)$, where $n$ depends on temperature $T$ and is determined by $\partial F / \partial n=0$. In this picture the free energy $F_{\alpha}(T)$ of the system with reduced entropy at temperature $T$ has the same properties as the original system at a lower temperature $\alpha T$. Thus if the original system had a roughening temperature $T_{R}$ the transition temperature $\widetilde{T}_{R}$ of the system with reduced entropy will increase to $\widetilde{T}_{R}$ $=T_{R} / \alpha$.

\section{E. Line energy versus step interaction}

As mentioned in the model definition, the ratio of line energy and elastic step interaction strength, i.e., the ratio $w_{1} / w_{\text {el }}$, can be varied by changing $p_{\max }$. Increasing $p_{\max }$, the self-interaction of the dipole charges becomes more and more important, whereas the long-range contributions lose significance. Due to its discrete nature, in the limit $p_{\max }$ $\rightarrow \infty$ our model becomes strictly local.

The line energy $w_{1}$ can be considered as a kind of effective $J_{\text {eff }}[$ see Eq. (9)] in comparison to purely local models, and in purely local models the roughening temperature is proportional to the only energetic scale $k_{\mathrm{B}} T_{\mathrm{R}} \sim J$. The dependence of the quantity $k_{\mathrm{B}} T_{\mathrm{R}} / w_{1}$ on $w_{1} / w_{\mathrm{el}}$ can therefore tell whether long-ranged elastic effects are important, or whether the model corresponds effectively to a local model with a coupling constant $J_{\text {eff }} \sim w_{1}$.

Computed results for infinite cutoff and $p_{\max }=0.336,1$, 6.39, 17.1, and 38.7 can be seen in Fig. 10. This series increases the line energy by factors of two. For large $w_{1} / w_{\mathrm{el}}$ the graph should approach the local limit. From simulations using $\Psi_{1,0}$, avoiding the numerically problematic limiting procedure $p_{\max } \rightarrow \infty$, we obtain $k_{\mathrm{B}} T_{\mathrm{R}} / w_{1} \approx 2.1$ using the criterion as in Fig. 4, which differs by about $10 \%$ from the more consistent value 1.9 derived by asymptotic scaling. The inset in Fig. 10 suggests that corrections to the asymptotic value behave as

$$
\left(k_{\mathrm{B}} T_{\mathrm{R}} / w_{1}-1.9\right) \sim 5.5\left(\frac{w_{1}}{w_{\mathrm{el}}}\right)^{-1} .
$$

The crossover computed from the intersection of the two asymptotics lies somewhere near $w_{1} / w_{\mathrm{el}} \approx 3$. Whenever the line energy is much larger than the amplitude of the step-step interaction term, the influence of step interaction on the roughening temperature can be neglected. If, however, $w_{1}$ is smaller than the step interaction amplitude, the long-range character of the elastic interaction is important and results in a strong increase of $k_{\mathrm{B}} T_{\mathrm{R}} / w_{1}$.

We would like to give an example of how the ratio $w_{1} / w_{\mathrm{el}}$ can be related to quantities measured in experiments or cal- 


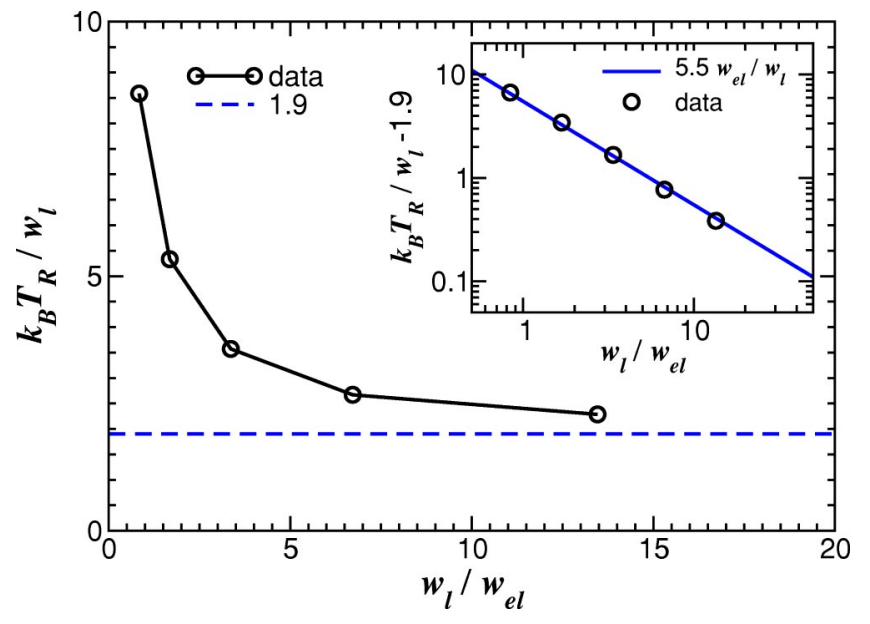

FIG. 10. Roughening temperature scaled by line energy $k_{\mathrm{B}} T_{\mathrm{R}} / w_{1}$ vs line energy scaled by elastic interaction strength $w_{1} / w_{\mathrm{el}}$. The scaled roughening temperature decreases with higher line energy as the model becomes effectively more local. The inset shows deviations of the scaled roughening temperature from asymptotics on a logarithmic scale.

culated from atomistic models. Usually ${ }^{25,26}$ the bare line or step energy $\beta^{0}$ without interaction is given in $\mathrm{eV} / \AA$, which can be related to the $w_{1}$ used here via $w_{l}=\beta^{0} a$. The strength of the elastic step-step repulsion can be extracted from the terrace width distribution (TWD) measured for a vicinal surface. ${ }^{27}$ Many authors assume an elastic interaction per length $A / d^{2}$ and present a value for the coefficient $A$ given in $\mathrm{eV} \AA$ as a result. Recalling Eq. (14) we can identify $8 w_{\mathrm{el}}$ $=A / a$, which leads to the result $w_{1} / w_{\mathrm{el}} \sim 8 \beta^{0} a^{2} / A$. Just to give the reader an idea about the order of this ratio we insert results for steps on $\mathrm{Si}(111)$ from a calculation based on empirical potentials. From Ref. 25 we conclude that $\beta^{0}$ is of the order $\beta^{0}=0.2 \mathrm{eV} / \AA$ and that the elastic interaction strength $A$ is of the order $A=0.2 \mathrm{eV} \AA$. With a lattice parameter of about $a \sim 5 \AA$ we end up with $w_{1} / w_{\mathrm{el}} \sim 2 \times 10^{2}$. On the other hand, Ref. 20 presents values for certain step configurations on $\mathrm{Si}(001)$, from which one could compute $w_{1} / w_{\mathrm{el}} \sim 2$. These results are arguable, since complicated effects such as surface reconstruction may interfere. But at least it indicates that there are probably materials where this ratio is small and the influence of long-range elastic effects cannot be ignored.

\section{CONCLUSION}

In summary, we have presented a model that contains the essential effects of long-range elastic repulsion between elastic defects on a crystal surface. We conclude that correlations due to these long-range interactions can strongly increase the roughening temperature in solid-on-solid models, mainly by a reduction of the entropy. Since defects prefer to be situated in secluded areas, the number of favorable configurations and consequently the entropy contribution to the free energy is diminished, leading to an increase of the roughening temperature. A scaling law, Eq. (23), has been found describing the change of the roughening temperature depending on the ratio of local and long-range energetic scales. Our simulations suggest that the type of transition remains the same, although a rigorous proof lies beyond the scope of this type of Monte Carlo approach.

\section{ACKNOWLEDGMENTS}

The authors would like to thank V.I. Marchenko for valuable comments and suggestions.
${ }^{1}$ J. M. Kosterlitz and D. J. Thouless, J. Phys. C 6, 1181 (1973).

${ }^{2}$ S. T. Chui and J. D. Weeks, Phys. Rev. B 14, 4978 (1976).

${ }^{3}$ J. M. Kosterlitz, J. Phys. C 7, 1046 (1974).

${ }^{4}$ J. M. Kosterlitz, J. Phys. C 10, 3753 (1977).

${ }^{5}$ T. Ohta and K. Kawasaki, Prog. Theor. Phys. 60, 365 (1978).

${ }^{6}$ H. J. F. Knops and L. W. J. Ouden, Physica A 103, 597 (1980).

${ }^{7}$ A. Prasad and P. B. Weichmann, Phys. Rev. B 57, 4900 (1998).

${ }^{8}$ P. J. M. Bastiaansen and H. J. F. Knops, Phys. Rev. B 53, 126 (1996).

${ }^{9}$ E. Jagla, S. Prestipino, and E. Tosatti, Phys. Rev. Lett. 83, 2753 (1999).

${ }^{10}$ J. D. Noh and M. den Nijs, J. Phys. A 30, 7375 (1997).

${ }^{11}$ D. L. Woodraska and J. A. Jaszczak, Phys. Rev. Lett. 78, 258 (1997).

${ }^{12}$ S. Prestipino, G. Santoro, and T. Erio, Phys. Rev. Lett. 75, 4468 (1995).

${ }^{13}$ M. den Nijs, Phys. Rev. Lett. 64, 435 (1990).

${ }^{14}$ G. Mazzeo, G. Jug, A. C. Levi, and E. Tosatti, Phys. Rev. B 49, 7625 (1994).

${ }^{15}$ A. Andreev and Y. Kosevich, Zh. Éksp. Teor. Fiz. 81, 1435 (1981) [Sov. Phys. JETP 54, 761 (1981)].
${ }^{16}$ V. Marchenko and A. Parshin, Zh. Éksp. Teor. Fiz. 79, 257 (1980) [Sov. Phys. JETP 52, 129 (1980)].

${ }^{17}$ J. Hardy and R. Bullough, Philos. Mag. 15, 237 (1967).

${ }^{18}$ V. M. Kaganer and K. H. Ploog, Phys. Rev. B 64, 205301 (2001).

${ }^{19}$ T. W. Poon, S. Yip, P. S. Ho, and F. F. Abraham, Phys. Rev. Lett. 65, 2161 (1990).

${ }^{20}$ T. W. Poon, S. Yip, P. S. Ho, and F. F. Abraham, Phys. Rev. B 45, 3521 (1992).

${ }^{21}$ J. Steinbrecher, H. Müller-Krumbhaar, E. Brener, C. Misbah, and P. Peyla, Phys. Rev. E 59, 5600 (1999).

${ }^{22}$ F. Gutheim, H. Müller-Krumbhaar, and E. Brener, Phys. Rev. E 63, 041603 (2001).

${ }^{23}$ J. Lapujoulade, Surf. Sci. Rep. 20, 191 (1994).

${ }^{24}$ Y. Saito and H. Müller-Krumbhaar, Phys. Rev. B 23, 308 (1981).

${ }^{25}$ S. Kodiyalam, K. Khor, N. Bartelt, and S. Das Sarma, Phys. Rev. B 51, 5200 (1995).

${ }^{26}$ G. Schulze Icking-Konert, M. Giesen, and H. Ibach, Phys. Rev. Lett. 83, 3880 (1999).

${ }^{27}$ T. Einstein, H. L. Richards, S. D. Cohen, and O. Pierre-Louis, Surf. Sci. 493, 460 (2001). 